\title{
HIGH-RESOLUTION ACQUISITION METHODS FOR WIDEBAND COMMUNICATION SYSTEMS
}

\author{
Irena Maravić ${ }^{\dagger}$, Martin Vetterli ${ }^{\dagger \dagger}$ and Kannan Ramchandran ${ }^{\ddagger}$ \\ ${ }^{\dagger}$ Audio-Visual Communications Laboratory \\ Swiss Federal Institute of Technology in Lausanne, CH-1015 Lausanne, Switzerland \\ ${ }^{\dagger \ddagger}$ Department of EECS, University of California at Berkeley, Berkeley CA 94720, USA
}

\begin{abstract}
We consider the problem of low-complexity timing synchronization in digital receivers for DS-CDMA and UWB systems operating over channels with single or multiple propagation paths. We extend some of our recent sampling results for certain classes of non-bandlimited signals and develop a method that takes advantage of transform techniques to perform propagation delay estimation from a lowdimensional subspace of a received signal, that is, by sampling below the traditional Nyquist rate. By lowering the sampling rate we reduce computational requirements compared to existing solutions, allow for slower A/D converters and significantly reduce power consumption of digital receivers.
\end{abstract}

\section{INTRODUCTION}

Synchronization in wideband communication systems is a crucial task that imposes serious restrictions on the system performance. There is a vast literature that has appeared recently, addressing both algorithmic and implementation issues of various synchronization techniques, with a clear trend to eliminate as much as possible the necessity for ana$\log$ components and perform all processing digitally ${ }^{1}$. Even though many high-performance schemes have already been proposed [1], their application in real time systems is often not feasible due to their high computational complexity. Furthermore, almost all of them use the Nyquist sampling rate, which requires very fast and expensive A/D converters and therefore high power consumption. This problem becomes critical in ultra-wideband systems, where in digitaloriented solutions $\mathrm{A} / \mathrm{D}$ converters must operate in the gigahertz range.

In this paper, we propose a low-complexity timing synchronization method which uses low-rate uniform sampling and well developed algorithmic solutions. We extend some

\footnotetext{
${ }^{1}$ Digital implementation offers many advantages, such as lower power, cheaper technology, full integration, robustness etc.
}

of our recent sampling results for certain classes of nonbandlimited signals [4] [6] to the problem of multipath delay estimation in wideband channels and develop an algorithm for estimating unknown propagation delays from a low-dimensional subspace of a received signal. Our approach leads to reduced computational requirements and lower power consumption compared to existing techniques, thus allowing for a practical hardware implementation and all the benefits of a digital design.

\section{SUBSPACE TIMING SYNCHRONIZATION}

\subsection{Problem Statement}

A number of propagation studies for wideband signals have been done, which take into account temporal properties of a channel or characterize a spatio-temporal channel response [2]. A common model for an impulse response of an outdoor multipath fading channel is

$$
h(t)=\sum_{l=1}^{L} a_{l} \delta\left(t-t_{l}\right)
$$

where $t_{l}$ denotes a signal delay along the $l$-th path while $a_{l}$ is a complex propagation coefficient which includes a channel attenuation and a phase offset along the $l$-th path. While this model does not adequately reflect specific bandwidthdependent effects, it provides a good characterization of the propagation channel used for diversity reception schemes in wireless cellular systems. Equation (1) can be interpreted as saying that a received signal $y(t)$ is made up of a weighted sum of attenuated and delayed replicas of a wideband transmitted signal $s(t)$, i.e.

$$
y(t)=\sum_{l=1}^{L} a_{l} s\left(t-t_{l}\right)+\eta(t)
$$

where $\eta(t)$ denotes receiver noise. Note that the received signal $y(t)$ has only $2 L$ degrees of freedom, time delays $t_{l}$ 
and propagation coefficients $a_{l}$, which can be efficiently estimated if we consider the problem in the frequency domain.

Let $Y(\omega)$ denote the Fourier transform of the received signal

$$
Y(\omega)=\sum_{l=1}^{L} S(\omega) a_{l} e^{-j \omega t_{l}}+N(\omega)
$$

where $S(\omega)$ and $N(\omega)$ are Fourier transforms of $s(t)$ and $\eta(t)$ respectively. Clearly, spectral components are given by a sum of complex exponentials, where the unknown time delays appear as complex frequencies while propagation coefficients appear as unknown weights. Thus the problem of estimating the channel parameters can be considered as a special case of classic harmonic retrieval problems that are well studied in spectral estimation literature [5]. There is a particularly attractive class of SVD-based algorithms, called super-resolution methods, which can resolve closely spaced sinusoids from a short record of noise-corrupted data. In the following, we will use a state space approach [5] that provides an elegant and numerically robust tool to estimate the channel parameters.

\subsection{Algorithm outline}

Our algorithm can be summarized as follows:

- Find the DFT coefficients $Y[m], m \in[-L, L]$, of $y(t)$ by sampling the signal with the sinc sampling kernel $\varphi(t)$ of bandwidth $\left[-L \omega_{0}, L \omega_{0}\right]$.

- Define a $P \times Q$ matrix $J$ as

$$
J=\left(\begin{array}{cccc}
Y_{s}[0] & Y_{s}[1] & \ldots & Y_{s}[Q] \\
Y_{s}[1] & Y_{s}[2] & \ldots & Y_{s}[Q+1] \\
\vdots & & & \\
Y_{s}[P] & Y_{s}[P+1] & \ldots & Y_{s}[P+Q+1]
\end{array}\right)
$$

where $Y_{s}[m]=Y[m] / S[m]$ and $P, Q \geq L$.

- Compute the singular value decomposition of $J$

$$
J=U \Lambda V^{H}+U^{\prime} \Lambda^{\prime} V^{\prime} H
$$

- Estimate the signal poles $z_{l}=e^{-j \omega_{0} t_{l}}$ as eigenvalues of a matrix $Z$

$$
Z=\underline{V}^{+} \cdot \bar{V}
$$

where $\overline{(\cdot)}$ and $\underline{(\cdot)}$ denote the operations of omitting the first and the last row of $(\cdot)$ respectively, while $(\cdot)^{+}$ denotes the pseudoinverse of $(\cdot)$.

- Find the propagation coefficients $a_{l}$ from the Vandermonde system

$$
Y_{s}[m]=\sum_{l=1}^{L} a_{l} e^{-j m \omega_{0} t_{l}}, \quad m \in[1, L]
$$

Note that in the above algorithm, we used a lowpass approximation of the signal to estimate all the relevant parameters. In general, the best performance of our method is achieved if we use a frequency band where the power spectral density of the signal is highest. Also note that we have converted the nonlinear estimation problem into the simpler problem of estimating the parameters of a linear model, and this using the shift invariance property of the matrix $J$. Nonlinearity is postponed for the last step, where in all spectral estimation methods the desired information is extracted from the estimated signal poles.

The computational requirement of the algorithm is dominated by the singular value decomposition part, which results in the overall computational order of $O\left(L^{3}\right)$. An alternative approach is to avoid SVD and estimate the signal parameters using the annihilating filter method [6], which has computational order of $O\left(L^{2}\right)$ and is particularly suitable for high and medium signal-to-noise ratios. The same framework can be extended to multiuser communication systems as well as joint angle and delay estimation using multiple antennas at the receiver [4].

\subsection{Synchronization of UWB signals}

Ultra-wideband (UWB) technology has recently received much attention due to benefits of exceptionally large fractional bandwidth [2], such as very fine time resolution for accurate ranging, imaging and multipath fading mitigation. UWB signals are generated by driving an antenna with very short electrical pulses, typically on the order of a nanosecond, thus spreading the signal energy from near DC to a few gigahertz. As a result, signal components propagating along different propagation paths undergo different frequency selective distortion and a more realistic channel model for UWB systems is of the form

$$
h(t)=\sum_{l=1}^{L} a_{l} p_{l}\left(t-t_{l}\right)
$$

with different pulse shapes $p_{l}(t)$ corresponding to different propagation paths. In this case, the DFT coefficients of the received signal are given by

$$
Y[m]=S[m] \sum_{l=1}^{L} P_{l}[m] a_{l} e^{-j m \omega_{0} t_{l}}+N[m]
$$

where $P_{l}[m]$ are now unknown coefficients. One possible way to jointly estimate the relevant channel parameters is to use an approximation of the spectral coefficients $P_{l}[m]$ with polynomials of degree $d \leq R-1$, i.e.

$$
P_{l}[m]=\sum_{r=0}^{R-1} p_{l, r} m^{r}
$$


Equation (9) now becomes

$$
Y[m]=S[m] \sum_{l=1}^{L} a_{l} \sum_{r=0}^{R-1} p_{l, r} m^{r} e^{-j m \omega_{0} t_{l}}+N[m]
$$

We can use the method from [6] and solve for an annihilating filter $H(z)$ (having zeros at $z_{l}=e^{-j \omega_{0} t_{l}}$ ) from the set of at least $2 R L$ DFT coefficients $Y[\mathrm{~m}]$

$$
H(z)=\prod_{l=1}^{L}\left(1-e^{-j \omega_{0} t_{l}} z^{-1}\right)^{R}
$$

Once the time delays have been estimated by finding the roots of $H(z)$, the polynomial coefficients $p_{l, r}$ can be obtained by solving the Vandermonde system (9). For a more detailed discussion on the annihilating filters, see [6]. It is interesting to note, however, that the propagation delays $t_{l}$ can be estimated with high accuracy by plugging the coefficients $Y[m]$ directly into (4), due to robustness of the state space approach to model mismatch. The estimated delays can be then used to find the coefficients $a_{l}$ and $p_{l, r}$.

\subsection{Timing recovery in UWB localizers}

An especially appealing application of our framework is in UWB systems used for precise position location. Such UWB transceivers, called localizers, have already been developed [3] and they use low duty-cycle episodic transmission of a coded sequence of impulses to ensure low power operation and good performance in a multipath environment. Yet, rapid sequence acquisition still presents a bottleneck in the current receiver design. For example, a solution proposed by Aetherwire [3] uses a cascade of 32 analog correlators and exhaustive search through all possible code positions. A major drawback of this approach, apart from being analog, is that it is inherently time consuming ${ }^{2}$ since transmissions are spaced apart while the correlation window spans a small fraction of the sequence cycle time $T_{\text {cycle }}$.

Our previous results can be directly extended to this case by modeling the received signal $y(t)$ as a convolution of $L$ delayed (possibly different) impulses with a known coding sequence $g(t)$

$$
y(t)=\sum_{l=1}^{L} a_{l} p_{l}\left(t-t_{l}\right) * g(t)
$$

while the correponding DFT coefficients $Y[\mathrm{~m}]$ are given by

$$
Y[m]=\sum_{l=1}^{L} a_{l} P_{l}[m] G[m] e^{-j m \omega_{c} t_{l}}, \quad \omega_{c}=2 \pi / T_{\text {cycle }}
$$

\footnotetext{
${ }^{2}$ It takes approximately 1000 cycles to acquire synchronization.
}

where $G[m]$ denote DFT coefficients of $g(t)$. If we use the polynomial approximation (10) of the spectral coefficients $P_{l}[m]$, the total number of degrees of freedom per one cycle is $2 R L$. Thus the signal parameters can be estimated using low-rate uniform sampling and the method we already described. This potentially leads to a few orders of magnitude faster acquisition, as will be shown in the next section.

\section{PERFORMANCE ANALYSIS}

We present simulation examples that illustrate the performance of our developed algorithm. We first consider the timing synchronization performance of a DS-CDMA system with BPSK modulation in an outdoor AWGN channel, where spreading is achieved with pseudo-random sequences of length 511. Figure 1 shows an average delay estimation error (normalized to the chip duration $T_{c}$ ) versus the sampling rate, and that for both non-fading channels 1(a) and multipath fading channels with 3 closely spaced paths 1(b). Our method clearly yields high resolution estimates, for example, by sampling the received signal at one fourth of the chip rate, the estimation error is less than one tenth of the chip duration (for $E_{b} / N_{0} \geq 7 \mathrm{~dB}$ ). The average error is somewhat higher for multipath channels, however, if we estimate the delay along the dominant path only (assuming there is one strongest component corresponding to a direct path), the algorithm performance is basically the same as in the non-fading channels.

UWB timing performance in a low duty-cycle regime is illustrated in Figure 2. We assumed that a transmitter periodically sends a coded sequence of 1023 first-derivative Gaussian impulses, where the sequence duration spans only $10 \%$ of the cycle time $T_{\text {cycle }}$. We considered a multipath channel (8) with 8 closely spaced paths and one dominant (direct) path. While synchronization can be achieved by considering the set of samples taken uniformly over $T_{c y c l e}$, it is more sensible to have a "multiresolution approach", where we first obtain a rough estimate of the sequence timing and then perform precise delay estimation by sampling the received signal only within a narrow window. Besides, for very low SNR's, it is desirable to consider a set of samples averaged over several cycles, which effectively increases the processing gain and results in better estimation performance. For example, for $S N R=-20 d B$ and a sampling rate of one tenth the Nyquist rate $\left(N_{s}=N_{n} / 10\right)$, it takes less than 20 cycles to achieve coarse synchronization (Figure 2(a)) and about 50 cycles for precise timing (Figure 2(b)) with $N_{s}=N_{n} / 5$, which is clearly a significant improvement compared to the existing solution [3].

Another attractive feature of our scheme is good performance in the presence of a strong narrowband interference. Namely, UWB systems must contend with a variety of interfering signals from narrowband systems and build- 
ing high-Q notch filters on chip would be technically very difficult. Since our method solves the estimation problem in the frequency domain, we can simply exclude spectral components that belong to frequency bands of interfering signals, while the state space algorithm can be easily modified to handle missing blocks of data [5]. The performance of our estimator in the presence of 3 strong sinusoidal signals is illustrated in Figure 2(b).

\section{CONCLUSION}

We presented a high-resolution method for timing synchronization in DS-CDMA and UWB communication systems operating over multipath fading channels, where all the steps are carried out on a low-dimensional subspace of the received signal. Our approach takes advantage of the wellknow spectral estimation techniques, requires much lower sampling rate, and therefore lower complexity and power consumption compared to existing methods. Besides, it leads to standard computational procedures and allows for identification of more realistic channel models without resorting to complex algorithms. In effect, we presented a "fully digital" solution that offers a possibility for lowpower single-chip implementation.

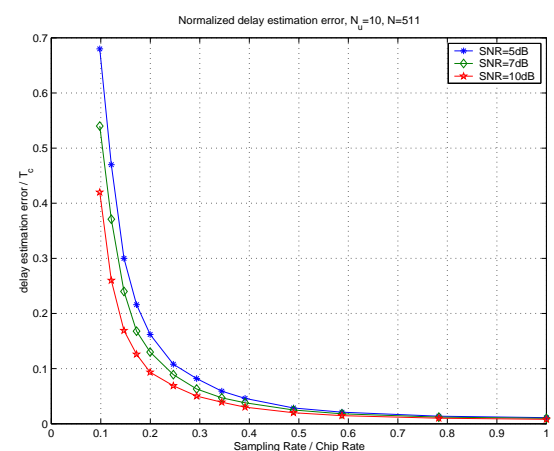

(a)

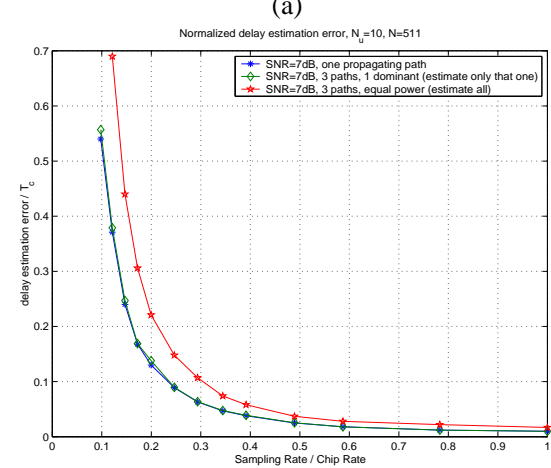

(b)

Fig. 1. Timing performance for CDMA signals (a) Timing synchronization error (normalized to $T_{c}$ ) in a non-fading channel vs. sampling rate.(b) Comparison of delay estimation errors in single-path and multipath channels ( 3 propagation paths, total delay spread is $10 T_{c}$ ).

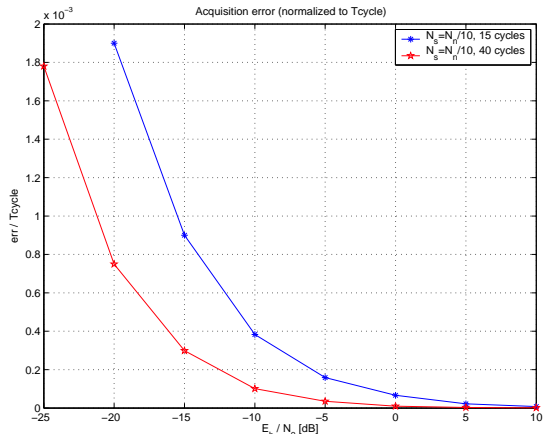

(a)

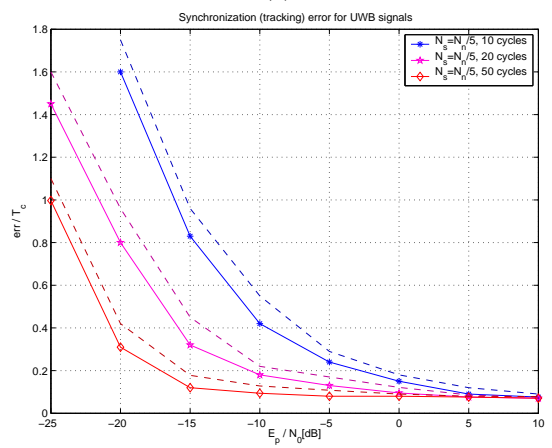

(b)

Fig. 2. Timing recovery in UWB systems (a) Acquisition error normalized to $T_{\text {cycle }}(\mathbf{b})$ Tracking error normalized to the chip time. Dotted lines: performance in the presence of 3 sinusoidal signals, $S I R=-35 \mathrm{~dB}$.

\section{REFERENCES}

[1] S. E. Bensley and B. Aazhang, "Maximum-Likelihood Synchronization of a Single User Code-Division Multiple-Access Communication Systems", IEEE Transactions on Communications, Vol. 46, No. 3., p.p. 392-399, March 1998.

[2] R. J. Cramer, R. A. Scholtz and M. Z. Win, "Evaluation of an Ultra-Wideband Propagation Channel", IEEE Trans. on Antennas and Propagation, Vol. 50, No. 5, pp 561-570, May 2002.

[3] R. Fleming, C. Kushner, "Spread Spectrum Localizers”, U.S. Patent 5,748,891, May 5, 1998.

[4] I. Maravić and M. Vetterli, "Digital DS-CDMA Receivers Working Below the Chip Rate", EPFL Technical Report IC/2002/018, also submitted to the IEEE Trans on Signal Processing, September 2002.

[5] B. D. Rao and K. S. Arun, "Model Based processing of Signals: A State Space Approach", Proceedings of the IEEE, Vol. 80, No. 2, pp. 283-309, February 1992.

[6] M. Vetterli, P. Marziliano and T. Blu, "Sampling signals with finite rate of innovation", IEEE Transactions on Signal Processing, June 2002. 